\title{
Soft Computing in Decision Support Systems
}

\author{
Matthew N. O. Sadiku ${ }^{1}$, Chandra M. M. Kotteti ${ }^{2}$, Abayomi Ajayi-Majebi ${ }^{3}$, and Sarhan M. Musa ${ }^{1}$ \\ ${ }^{1}$ Roy G. Perry College of Engineering \\ Prairie View A\&M University, Prairie View, TX, USA \\ ${ }^{2}$ School of Computer Science and Information Systems \\ Northwest Missouri State University, Maryville, MO 64468 \\ ${ }^{3}$ Department of Manufacturing Engineering \\ Central State University, P.O. Box 1004 \\ Wilberforce, OH, USA
}

\begin{abstract}
The complexity of modern decisions has caused organizations to become increasingly dependent on advanced intelligent technologies to quickly process large amounts of data and make informed decision. Decision support system (DSS) is a computerbased system that helps in the process of decision making. It is designed to support better-informed decision-making. It is used to support determinations, judgments, and courses of action in an organization. Intelligent decision support systems based on soft computing have attracted the attention of researchers and practitioners in a wide range of disparate areas from engineering to business. This paper is an introduction on the applications of soft computing in decision support system.
\end{abstract}

Key Words: Computer Science, Hard Computing, Decision Making, Decision Support System, Soft Computing,

\section{INTRODUCTION}

A decision maker is anybody who makes decisions or takes a part in a decision making process. We all make many decisions daily. Rapid advances in technology tends to make decision environments more and more complex. The emergence of the intelligent decision making technology provides a good decision support to deal with these complex decision making problems. Soft computing plays a core role in the intelligent decision making technology. In human decision reasoning, everything is a matter of degree: suitability, importance, simultaneity, substitutability, and similar indicators are all soft computing (fuzzy) values [1].

Decision support system (DSS) is a computer program application that supports business and organizational decision-making activities. It analyzes large amounts of data and presents a company with the best possible options available, thereby improving a company's decision-making capabilities. An ideal DSS analyzes information and allows human users to make more informed decisions at a quicker pace. Typical information used by a DSS includes projected revenue, sales figures, and other operationsrelated data. This information may be unstructured or semi-structured. An DSS is capable of integrating multiple variables and generate alternate outcomes, all based on the company's current and historical company data [2].

A typical DSS consists of three different parts as shown in Figure 1 and explained as follows [3]:

- Knowledge base containing information from both internal and external sources. It stores information used by the system's reasoning engine to determine a course of action.

- Management system which is composed of model management systems. Organizations use models to predict how outcomes will change with different adjustments to the system. 
- User interface which makes it easy for the user to manipulate the data that is stored on it. DSS interfaces include simple windows, complex menu-driven interfaces, and command-line interfaces.

Different kinds of decision support system can improve a company's decision-making capabilities in a variety of areas. Decision methodology can be based on soft computing and consistent with observable properties of human reasoning. Soft-computing approaches are applied in industries such as finance, retail, construction, healthcare, and agriculture to help with decision-making and problem-solving.

The term "soft computing" was coined by Lofti A. Zadeh in 1991. Since then, the area has experienced rapid development. Soft Computing became a discipline within computer science in the early 1990s. The terms "machine intelligence" and "computational intelligence" have been used to have close meaning as soft computing.

The principal premise of soft computing (SC) is that we live in a world that is imprecise and uncertain. Soft computing refers to the use of "inexact" solutions to computationally hard tasks [4]. Soft computing has provided advanced methodologies for the development of decision support systems.

\section{OVERVIEW OF SOFT COMPUTING}

Soft computing (SC) is a branch of computer science that resembles the processes of the human brain. It may also be regarded as a newly emerging multidisciplinary field. Its main objective is to develop intelligent machines in order to solve real- world problems. Soft computing differs from the conventional hard computing as it can handle uncertainty, imprecision, partial truth, and approximation. The role model for soft computing is the human mind. Soft computing techniques are iterative in nature and provide flexibility to handle many real-time, real-world problems. Figure 2 illustrates hard and soft computing approaches [5]

Soft computing refers to a collection of computational techniques in computer science, artificial intelligence, and machine learning. The techniques aim to exploit the tolerance of imprecision and uncertainty to achieve tractability, robustness, and low solution cost. Its principle components include:

- Expert systems

- Neural networks,

- Machine learning

- Probabilistic reasoning

- Evolutionary algorithms

- Artificial neural networks

- Fuzzy logic

- Swarm intelligence

- Interactive computational models

These computation methods or technologies provide information processing capabilities to solve complex practical problems. They should be considered complementary instead of competitive. Some of these techniques are illustrated in Figure 3 [5].

\section{APPLICATIONS OF SC IN DECISION SUPPORT SYSTEMS}

Soft computing is used for solving real-life problems and can be applied in different fields such as education, healthcare, business, economics, industry, engineering, power systems, transportation, communication systems, wireless communications, data mining, home appliances, robotics, etc. Several decision support systems have been developed mostly in different fields such as medical diagnosis, business management, control system, military, and agriculture. DSS can be built in almost any knowledge domain. Common applications of soft computing in DSS are presented as follows:

- Smart City Management: The transition from the traditional city to the smart city is made through achieving a more efficient and more responsible city. A city is regarded "smart" when investments in human and social capital all fuel efficient and sustainable economic development. Smart city is a wise management of natural resources. Smart Cities are the result of a dynamic process which develops along six dimensions: smart economy, smart people, smart mobility, smart environment, smart living, and smart governance. Several business organizations, academic institutions, and governments have invested significantly in the implementation of the smart city concept during the past decade. Decision support system (DSS) offers the strategic decision makers the necessary tools to plan and complete projects for the development of a smart city and smart home. The rapid evolution of technologies position DSS at a unique advantage as a continuously improving tool in the process of planning and completing complex projects [6].

- Clinical Decision Support Systems: This DSS is for medical diagnosis for various diseases such as cancer, liver fibrosis, etc. It is mainly used to consult and support medical professionals. Soft-computing approaches are applied in healthcare, 
particularly in the context of clinical decision support systems (CDSS). Such techniques can assist in critical decisionmaking, support diagnosis procedures, and consult medical professionals. Diagnosis of deadly diseases, such as liver fibrosis, is very important. Arriving at an accurate and timely diagnosis decision is always difficult due to dynamic, vagueness, and uncertainty associated with diseases. Accurate and timely medical diagnosis is critical in the healthcare industry because accuracy and timeliness directly impact the outcome of the treatment of a patient. It is not easy to determine the environment's effect on the epigenetics of a patient and, hence, its effect on the disease outcome. Thus, it becomes very time and energy consuming for medical practitioners to come to a conclusive diagnosis. CDSS is used by the medical professionals in diagnosis, prognosis, and prediction. Soft computing's applicability in CDSS has revolutionized the field of diagnosis and tracking of a patient's treatment. CDSS is a complex system that employs softcomputing techniques such as adaptive neuro-fuzzy inference system, artificial neural networks, and fuzzy cognitive maps [7,8]. For example, fuzzy cognitive map based decision support system for medical diagnosis can diagnose dyslexia (difficulty in learning). The combination of these methodologies may pave the way to new sophisticated systems.

- E-learning Decision Support: The fast increasing popularity of the Internet and the advance of telecommunication technologies is determining the next generation of distance education tools. Web-based education has revealed itself as a useful tool for course delivery and knowledge sharing. The Internet medium is used to convey content and allows the gathering of information on students' online behavior. One of the most challenging and time consuming activities for teachers in distance education is the evaluation process. An e-learning decision support system can be developed to allow the e-learning systems improvement of the learning experience. This framework is based on soft computing techniques. The goal of such a system is to help virtual teachers to understand the underlying relations between the actions of the learners and make more interpretable the student's learning behavior [9].

- Forecasting: Accurate forecasting is very important for many management decisions. Traditional forecasting techniques depend on proper specifications of systems. A statistical time series forecasting method is often applied. Many statistical methods have been used to forecast time series. Exponential procedures are widely used as forecasting techniques for inventory control and business planning. This requires the user to specify initial values for the level, trend, and seasonal components. Advances in data analysis and software capabilities have the potential to offer effective forecasting to anticipate future demands. Soft computing methodology can be used due to the imprecise knowledge of the goals and the need for managing several sources [10].

Other areas of application of soft computing in decision support system include agriculture, education, forest management, risk assessment, GPS route planning, fetal delivery, software project development, and forecasting.

\section{BENEFITS}

Changing decision-making environments, managerial requests, and decision-maker limitations create a need for more and better decision support. Many organizations have integrated computerized decision support into day-to-day operations. The primary purpose of using a DSS is to present information to the human users in an easy-to-understand way. A decision support system allows for more informed decision-making, timely problem-solving, and improved efficiency in dealing with issues or operations, planning, and even management. It provides data-driven recommendations that take the guesswork out of decision-making. It can be programmed to generate many types of reports. A DSS can be tailored for any industry, profession, or domain including the medical field, government agencies, agricultural concerns, and corporate operations. The flexible nature of the DSS is beneficial for users or customers on the go or even on the spot.

SC techniques can allow a DSS to have a level of decision automation that is more valuable than is possible without SC. When SC is used to produce a DSS, the DSS can provide greater productivity benefits to the decision process.

\section{CHALLENGES}

Some resist the idea of trusting complex computer software solutions to make decisions for them. It is challenging to determine which of several possibilities will maximize returns. It is also difficult to anticipate external changes that may impact profitability since companies are vulnerable to external influences, such as political uncertainty, major weather events, trade disputes, and changes in the global market. These factors may create a storm, where decision-making is hampered by a lack of predictability, as well as by an inability to process data fast enough to support decisions [11].

Efficiency requirements in developing specific methodologies and tools for each modeling paradigm has resulted in paradigm specific problem representations. As a consequence, it has become increasingly difficult to apply all pertinent paradigms to a problem at hand, because these resources are fragmented. Using more than one paradigm for a problem at hand is expensive and 
time consuming in practice. Models have become complex and large, making the development of a model a costly and timeconsuming process. Therefore, multi-paradigm modeling support is currently one of the most challenging problems the scientific community [12].

\section{CONCLUSION}

Rational decision making is becoming increasingly difficult. A decision support system (DSS) may be regarded as a computerized system that gathers and analyzes data to produce comprehensive information reports. DSS offers business a powerful tool that is rapidly becoming an indispensable component of managerial task. Intelligent decision support systems based on soft computing are of great importance to deal with complex decision environments. Soft Computing (SC) techniques are based on exploiting human knowledge and experience and they are extremely useful to model any complex decision making procedure. Soft computing approaches can deal with complex decision information such as uncertain and inconsistent information [13]. More information about soft computing in economics can be found in the books in $[14,15]$ and the following related journals:

- $\quad$ Soft Computing

- $\quad$ Applied Soft Computing

- International Journal on Soft Computing

- Journal of Soft Computing and Decision Support Systems

- Journal Soft Computing Exploration

\section{REFERENCES}

[1] J. Dujmovic and W. L. Allen III, "Soft computing logic decision making in strategic conservation planning for water quality protection," Ecological Informatics, vol. 61, March 2021.

[2] T. Segal, "Decision support system (DSS)," January 2022, https://www.investopedia.com/terms/d/decision-support-system.asp

[3] "What is a decision support system (DSS)?"

https://corporatefinanceinstitute.com/resources/knowledge/other/decision-support-system-dss/

[4] M. N. O. Sadiku, Y. Wang, S. Cui, S. M. Musa, "Soft computing: An introduction," International Journal of Advanced Research in Computer Science and Software Engineering, vol. 8, no. 6, June 2018, pp. 63-65.

[5] S. B. B. Priyadarshini et al., "A comprehensive review on soft computing framework," International Journal of Advanced Mechanical Engineering, vol. 8, no. 1 2018, pp. 221-228.

[6] V. Chichernea, "The use of decision support systems (DSS) in smart city planning and management," Journal of Information Systems \& Operations Management, vol. 8,no. 2, December 2014.

[7] B. Kukreja, H. Kaur, and A. Chowdhary, "Soft-computing approach in clinical decision support systems," in S. Dash et al. (eds.), Deep Learning, Machine Learning and IoT in Biomedical and Health Informatics. Boca Raton, FL: CRC Press, 2022.

[8] S. El-Sappagh et al., "Clinical decision support system for liver fibrosis prediction in hepatitis patients: A case comparison of two soft computing techniques," IEEE Access, vol. 6, 2018, pp. 52911-52929.

[9] F. Castro, A. Nebot, and F. Mugica, "A soft computing decision support framework to improve the e-learning experience", Proceedings of the 2008 Spring Simulation Multiconfrence, April 2998, pp. 781-788.

[10] J.D. Bermúdeza, J.V. Segurab, E. Verchera, "A decision support system methodology for forecasting of time series based on soft computing," Computational Statistics \& Data Analysis, vol.51, 2006, pp. 177 - 191.

[11] "Five decision support system examples you need to know,"

https://www.riverlogic.com>-

[12] M. Makowski et al., Applied Decision Support With Soft Computing. Springer, Undated.

[13] "Special issue on intelligent decision support systems based on soft computing," Applied Soft Computing, October 2016

[14] X. Yu and J. Kacprzyk (eds.), Applied Decision Support with Soft Computing. Springer 2003.

[15] Decision Making and Soft Computing: Proceedings of the 11th International FLINS Conference, Brazil, August 2014. 


\section{ABOUT THE AUTHORS}

Matthew N. O. Sadiku is a professor emeritus in the Department of Electrical and Computer Engineering at Prairie View A\&M University, Prairie View, Texas. $\mathrm{He}$ is the author of several books and papers. His areas of research interest include computational electromagnetics and computer networks. He is a fellow of IEEE. Email: sadiku@ieee.org

Chandra M. M. Kotteti received his M.S. degree in applied computer science from Northwest Missouri State University, MO, in 2014, and a Ph.D. degree in electrical engineering from Prairie View A\&M University, TX, in 2020. He currently works as an assistant professor in the School of Computer Science and Information Systems at Northwest Missouri State University, MO. His current research interests include machine learning, deep learning, data science, and computer science. Email: chandra@nwmissouri.edu

Abayomi Ajayi-Majebi is a professor in the Department of Manufacturing Engineering at Central State University in Wilberforce, Ohio. In 2015 he was honored by the White House as a Champion of Change for his significant contributions to the engineering education of minority students. He is a senior member of both the Society of Manufacturing Engineers and the American Society for Quality. Email: ajayi-majebi@ centralstate.edu

Sarhan M. Musa is a professor in the Department Electrical and Computer Engineering at Prairie View A\&M University, Texas. He has been the director of Prairie View Networking Academy, Texas, since 2004. He is an LTD Sprint and Boeing Welliver Fellow. His areas of research interest include computational electromagnetics and computer networks. Email: smmusa@pvamu.edu

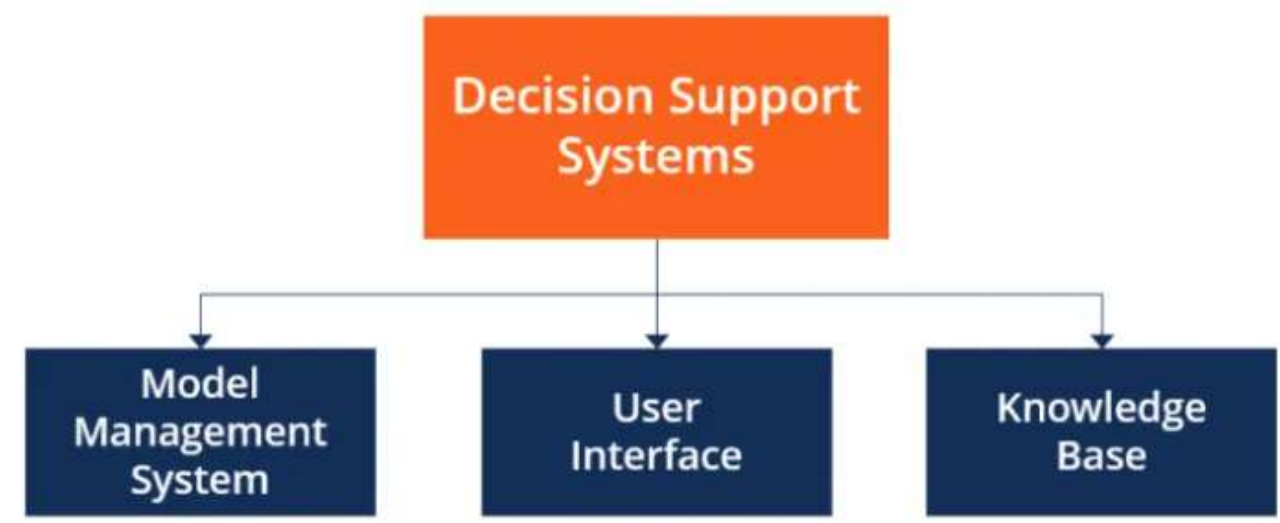

Figure 1 Three components of a decision support system [3]. 
Problem Solving Technology

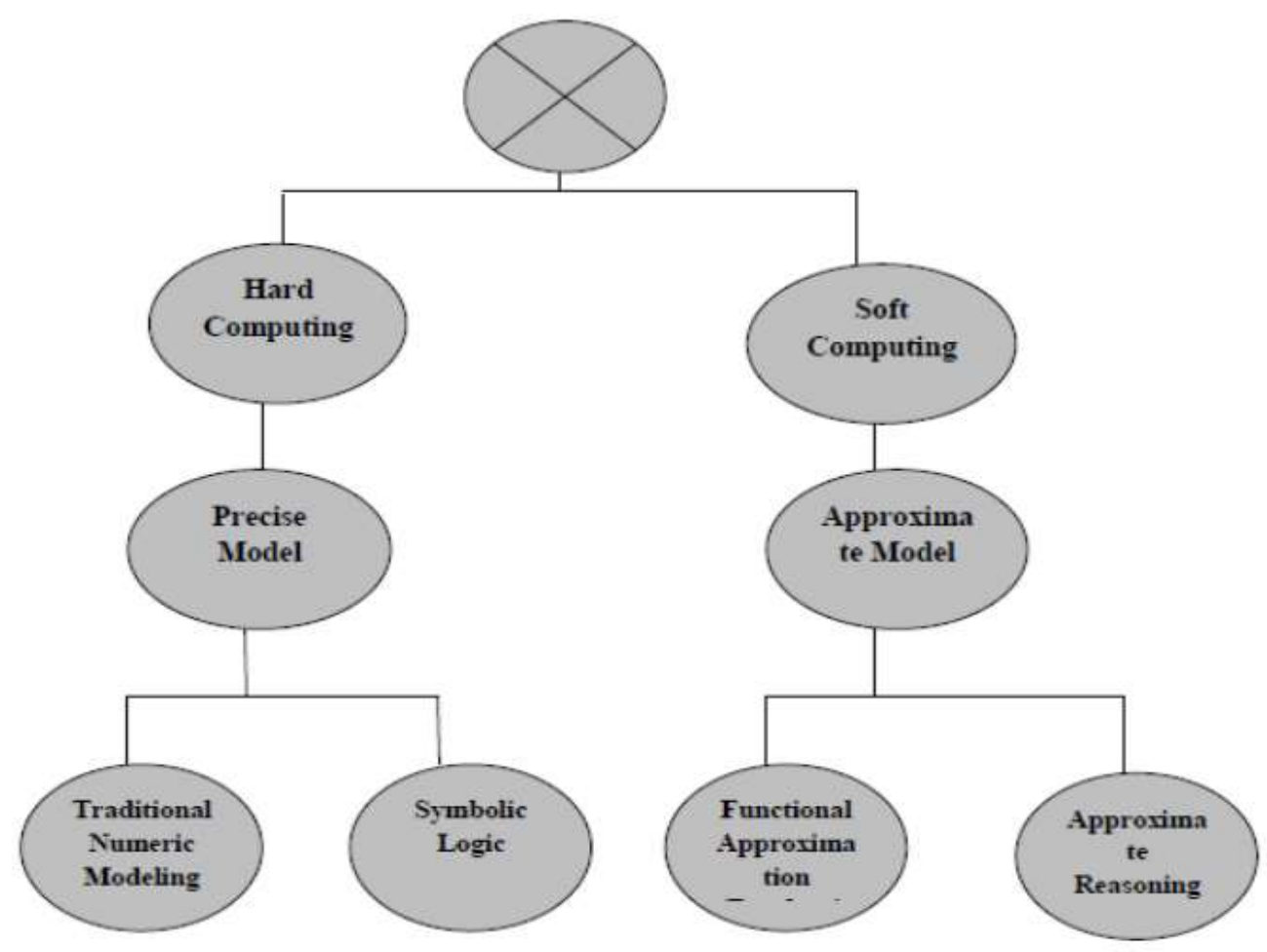

Figure 2 Hard and soft computing approaches [5].

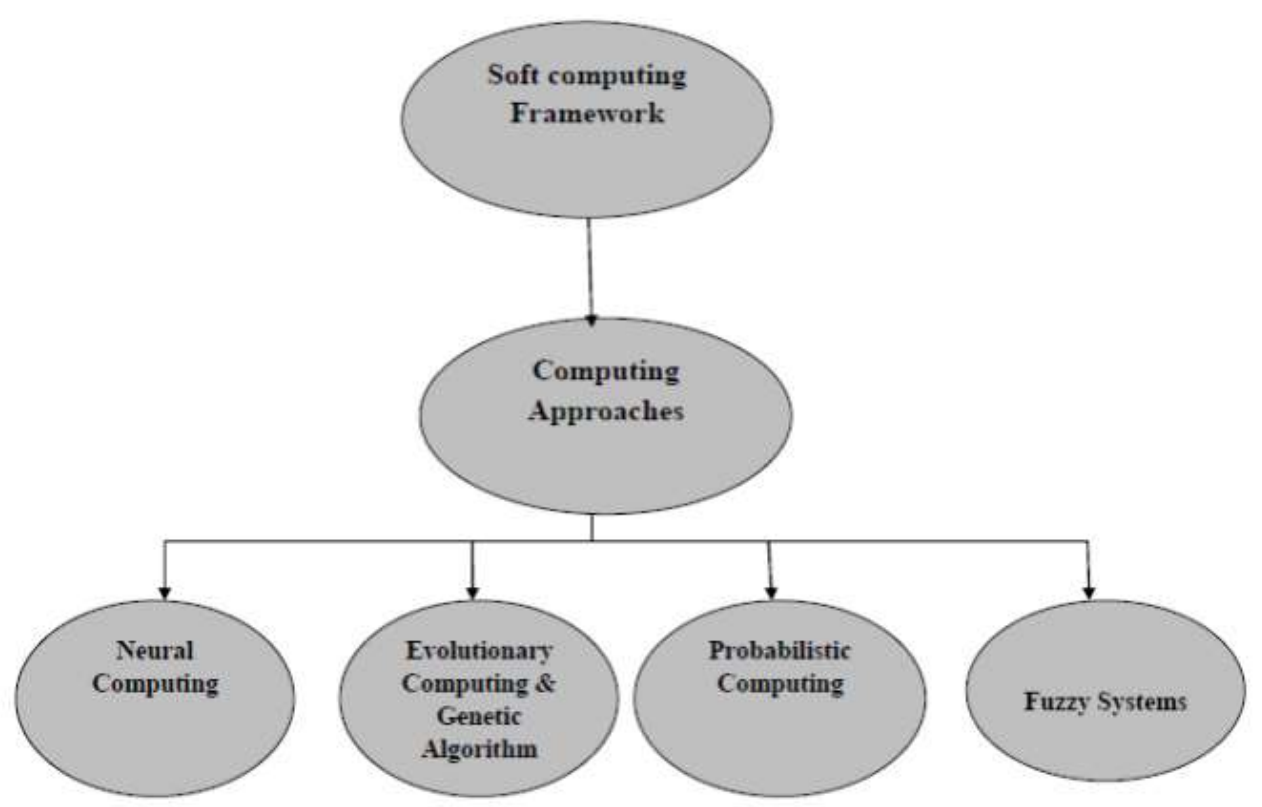

Figure 3 Soft computing techniques [5]. 